\title{
The Influence of Key Process Parameters on Melt Pool Geometry in Direct Energy Deposition Additive Manufacturing Systems
}

\author{
Robert Sampson ${ }^{1}$, Robert Lancaster ${ }^{2 *}$, Mark Sutcliffe ${ }^{1}$, David Carswell ${ }^{1}$, Carl Hauser ${ }^{1}$, Josh Barras ${ }^{1}$ \\ ${ }^{1}$ TWI Granta Park, Great Abington, Cambridge, CB21 6AL, UK, robert.sampson@ @twi.co.uk; \\ mark.sutcliffe@twi.co.uk; david.carswell@twi.co.uk; carl.hauser@twi.co.uk; josh.barras@twi.co.uk \\ ${ }^{2}$ Swansea University, Bay Campus, Fabian Way, Swansea, SA1 8EN, \\ *Corresponding Author: Robert Lancaster, r.j.lancaster@swansea.ac.uk, +441792295965
}

\begin{abstract}
The measurement of melt pool width has proven to be critical in the development of advanced control systems, defect detection algorithms and understanding the influence of parameter changes on build quality in additive manufacturing processes. Parametric studies are performed on new materials and builds to help identify optimal parameter settings for final components. Many parametric iterations are often required to produce optimal components, which requires in-depth process/material knowledge from highly skilled engineers. Having fundamental knowledge of parametric interaction and melt pool mechanics is key in developing state of the art additive manufacturing components. This paper addresses the need for complex parametric interaction understanding in additive manufacturing processes by providing a detailed parametric study using directional emittance melt pool edge detection techniques.
\end{abstract}

Keywords: Melt pool; melt pool monitoring; additive manufacturing; direct energy deposition; process parameters

\section{Introduction}

Additive Manufacturing (AM) is a progressive technology that is currently being utilised in multiple industries including the aerospace, automotive and medical sectors. AM is a family of manufacturing technologies that fabricate components by adding material, as opposed to conventional subtractive techniques such as milling and lathing. The umbrella term AM can be broken down into seven subcategories: vat photopolymerisation, material extrusion, material jetting, binder jetting, Powder Bed Fusion (PBF), Direct Energy Deposition (DED) and sheet lamination. DED is a powder blown technique that induces a laser beam onto a substrate and injects powder into the molten metal pool (melt pool) formed to enlarge it. Melt pools can be controlled by robotic manipulators to form complex near net shape structures. This paper focuses on the implementation of Melt Pool Monitoring (MPM) techniques into DED processes. Whilst specific focus has been placed on DED, it should be noted that MPM advancements made for DED systems can be transferred or adapted into PBF systems and vice versa.

MPM systems have been used in AM processes for many years to develop defect detection capabilities, control systems and better understand process parameters. A more holistic understanding of input parameters can benefit AM research organisations, as it allows for good manufacturing practices and optimum component quality.

Key process parameter knowledge allows skilled engineers to manually manipulate parameters to produce high quality components. Meriaudeau \& Truchetet (1996) determined key process variables by integrating multiple cameras into $\mathrm{PBF}$ systems to measure powder distribution, track surface temperature and track geometry. They determined that the Powder Mass Flow Rate (PMFR) affects track height and suggested that PMFR, laser power and beam diameter can affect bonding quality. Renier, Meriaudeau, \& Truchetet (1996) further studied the influence of welding temperature on track deposition using image processing techniques. This knowledge was used to optimise industrial weld applications, with their studies identifying melt pool temperature to be a key process parameter. 
Meriaudeau et al. (1997) subsequently developed image-based powder particle speed measurement techniques and identified Powder Mass Flow Rate (PMFR) as a key process variable that influences both the melt pool and AM process. Hua et al. (2008) developed a temperature measurement system using a two-colour infrared thermometer to investigate the influence of process parameters (including PMFR, scanning velocity and laser power) on melt pool temperature. They concluded that increasing PMFR decreases melt pool temperature, increasing scanning velocity decreases melt pool temperature and increasing laser power increases melt pool temperature. Sun \& Hao (2012) studied the influence of changing PMFR, laser power and scanning speed on both track geometry and dilution (the relation between the melted area of the substrate and the amount of added material). They discovered that PMFR is the dominant factor that affects both the width and height of the track, whilst scanning speed has the most effect on molten depth of substrate. Cheng et al. (2018) studied the influence of changing path velocity on melt pool geometry and identified it as a key process variable that influences both melt pool and component. They found that increasing path velocity results in a decrease in melt pool width. The effects of thermal behaviour, or the evolution of temperature during PBF, on the melt pool and part quality is studied in detail by Yan et al. (2018). They concluded that the thermal behaviour from the process and melt pool mechanics influence residual stress, deformation, microstructure and defects. Yao et al. (2018) performed a parametric study using a DED system and identified that laser energy density is positively correlated to part density. They found that the key parameters to influence energy density are laser power, path velocity and PMFR.

Melt pool behaviour is influenced by many parameters within AM processes. Hooper (2018) studied the effects of geometry and scan path on the melt pool's temperature gradients and cooling rates. Hooper determined that events such as beginning scans, ending scans and turning during hatching affect temperature gradients, cooling rates and melt pool stability. Hooper's results displayed fluctuations in both temperature gradients and cooling rates during multiple events. Hooper concluded that a deeper understanding of process fundamentals can accelerate and refine process parameter development to provide improved component quality. Craeghs et al. (2012) studied changes in melt pool behaviour with overhanging structures. They discovered a novel way of presenting thermal data and determined that overhang structures (geometry) can have a major influence on the melt pool. Results from Craeghs et al. (2012) show that increasing the amount of support structures on layers prior to building overhanging features causes more uniform heat dissipation and reduced overheating of powder, due to the supports acting as a heat sink. Kledwig et al. (2019) used an MPM system in a DED process to identify the minimum energy required to produce stable melt pools. They used optical monitoring systems and a pixel counting method to identify the melt pool area. The study was able to determine influences of laser power, PMFR and path velocity on melt pool area. It identified parametric boundaries for the material X2CrNiMol7-12-2 such as the minimum required specific energy $\left(18 \mathrm{~J} / \mathrm{mm}^{2}\right)$. Furumoto et al. (2018) used high-speed imaging and studied the influence of surface temperature and build sequence on resultant formation and spatter particles. They determined that substrate temperature influences melt pool behaviour and spatter patterns. Manvatkar and DebRoy (2015) created a three-dimensional transient heat transfer and fluid flow model to determine changes in melt pool geometry and cooling rates across different heights layers in build cycles. They determined that peak temperature and melt pool dimensions increase and cooling rates decrease as build layers are added.

Melt pool behaviour has been linked with both process parameters and the integrity of the manufactured component. Direct input parameters such as laser power, PMFR and path velocity can be used to influence the melt pool and improve part quality. Dai et al. (2018) identified a new scan strategy to greatly improve component quality. They increased part density and improved tensile properties by remelting previous layers using a higher laser volume energy density. This liberated entrapped gas, promoting the formation of high densification. Fiegl, Franke, and Körner (2019) established that the position and orientation of components within build chambers influences surface roughness, porosity levels and tensile strength of built parts. They discovered that increasing the distance between the component and zero-point (directly below the laser), resulted in an increased surface roughness and porosity, and decreased tensile strength and elongation. Ding et al. (2019) studied the process parameters from the Electron Beam Melting (EBM) process and successfully linked resultant microstructure, melt pool geometry and input parameters together. They discovered that increasing line 
energy resulted in an increase in melt pool width and depth. During this process they identified that the shape of the melt pool progressed from semi-elliptical to semi-circular and then to conical. Colodrón et al. (2011a) measured melt pool width using emissivity-based image processing techniques and determined it to be a good indication of process status. They discovered that melt pool width could be correlated to dilution. In a subsequent study, Colodrón et al. (2011b) then used melt pool width calculations to improve part quality by creating a control system to regulate laser power. They found that control systems are useful for components with complex geometries, where individual cladding tracks are non-continuous and short. A similar technique was developed by Hofman et al. (2012), who successfully used a CMOS camera and software to calculate melt pool geometry. They regulated melt pool width by using a control system to alter laser power throughout the build. They discovered that a constant laser power leads to excessive dilution and reduced hardness. By controlling the laser power, and thus melt pool width, they reduced dilution in DED processes. Sun, Guo, and $\mathrm{Li}$ (2020) developed a melt pool dimensioning system that can accurately predict melt pool width and shape in DED processes. They discovered that melt pool boundary shape can be used to predict the crystal growth direction in manufactured components.

Research has shown that MPM systems can be used in a range of applications to improve AM processes and components. This research aims to utilise a previously documented melt pool dimensioning technique, developed by Sampson et al. (2020), to better understand process parameters for subjective build optimisation decisions.

This study focuses on the influence of two major parameters in DED processes - PMFR and path velocity. These parameters have been selected from experience of DED processes and are supported by literature. Meriaudeau et al. (1997) identified PMFR as a major influence on the melt pool and process. Cheng et al. (2018) studied the influence of path velocity on melt pool width and identified it as a parameter that influences both melt pool and component. Yan et al. (2018) provided a recent parametric study to identify both PMFR and path velocity as key processing parameters in DED processes.

PMFR and path velocity changes can have both positive and negative effects on melt pool stability and build quality. High speed deposition and low powder wastage can be achieved by optimising parameters. Manufacturing outside of a material's parametric envelopes however can cause melt pool instability, and defects such as lack of fusion and keyhole porosity. Often material parameter envelopes are not understood, and no guidance is provided for the distribution of powder material for DED processes. This study aims to utilise advanced MPM techniques by Sampson et al. (2020), to better understand the complex interaction of laser power, PMFR and path velocity parameters when manufacturing with the steel alloy, EN25.

\section{Melt Pool Monitoring System}

The MPM system used in this research has been previously disseminated in a paper by Sampson et al. (2020). The MPM system can provide accurate melt pool width calculations without the need for emissivity values. The technique uses improved machine vision techniques to highlight features that best indicate the true melt pool edge when depositing with EN25.

\section{Adaptive Exposure Times}

Adaptive exposure times are used to achieve high-quality melt pool images over a range of parameter settings. To calibrate exposure times for EN25, a series of scans were produced to identify the best exposure time for various laser power settings. The laser power and exposure times are correlated subjectively by the operator to increase the directional emittance phenomenon. The experimental procedure used to create Equation 1 is detailed within a paper previously disseminated by Sampson et al. (2020). Equation 1 is used to identify the optimum exposure time for laser power settings between $600-1200 \mathrm{~W}$.

$$
E=-6.0 \times 10^{-4} . P+0.9192
$$


Where $E$ is the exposure time setting to be used, and $P$ is the laser power setting being used when depositing EN25 powder. Figure 1 shows the melt pool images captured using optimum exposure times over multiple laser power settings. The images illustrate melt pool edge features and have low levels of saturation.
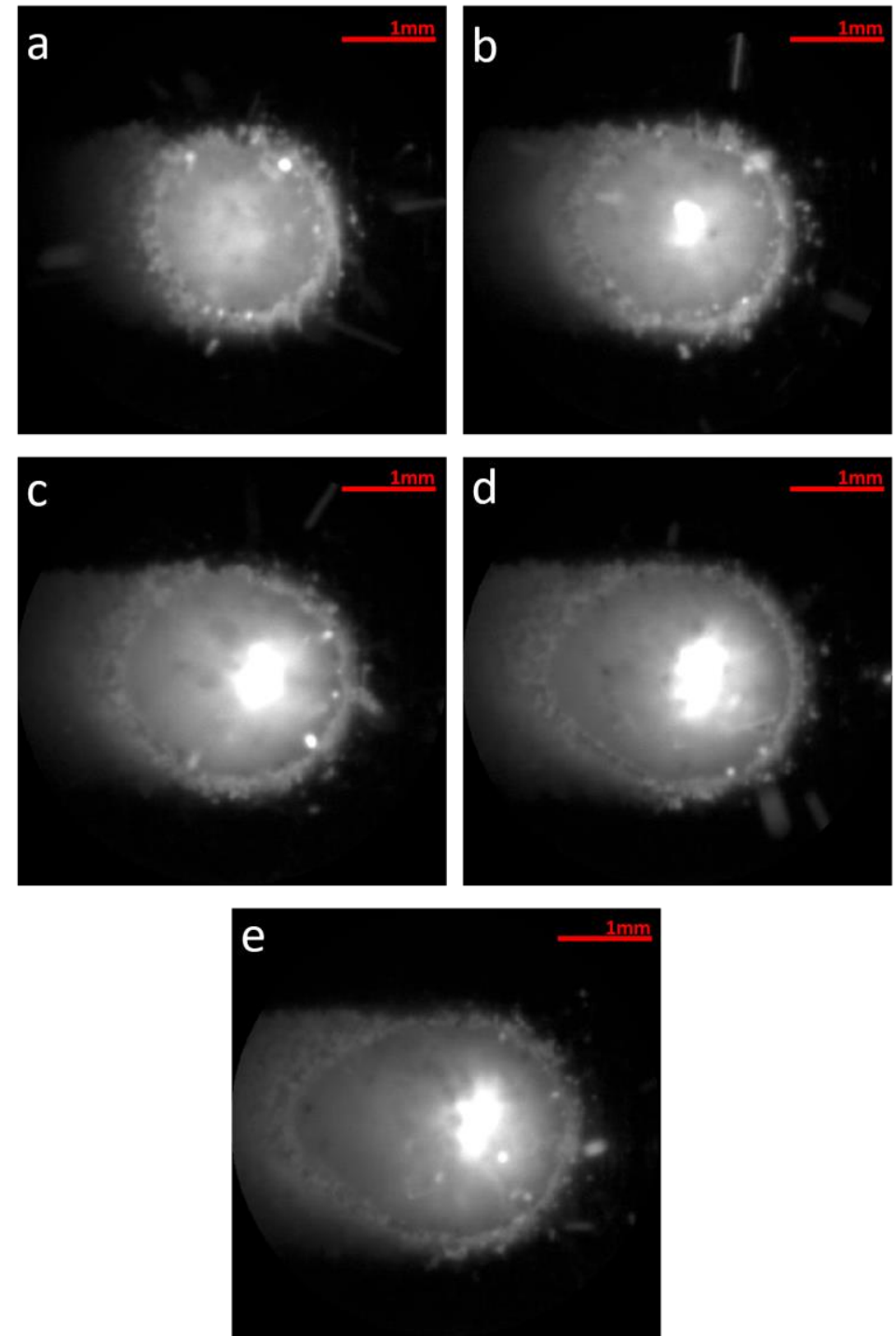

Figure 1. A series of melt pool images for various laser powers taken using adaptive exposure times. The laser power settings are: a) $600 \mathrm{~W}$ b) $750 \mathrm{~W}$ c) $900 \mathrm{~W} \mathrm{d)} 1050 \mathrm{~W}$ and e) $1200 \mathrm{~W}$.

\section{Directional Emittance Phenomena}

Emissivity describes a surface's ability to emit radiation. Emittance is the radiation mismatch between a real surface and a blackbody of that material. A blackbody is an idealistic surface that absorbs all incident light, independent of both wavelength and direction, and neither reflects nor transmits. Real surfaces almost never have properties of a blackbody. The surface of a real body is one that only partially emits radiation compared with a blackbody at the same temperature and wavelength. A real body does not emit radiation equally for all wavelengths in all directions.

As described by Meola (2016), the directional emittance phenomenon occurs in melt pool images when using optimised exposure times as a result of real body radiation not emitting equally in all directions. This results in part of the substrate emitting stronger radiation in the direction of the sensor even though the surface temperature is lower. 
Radiation emitted from the melt pool in the direction of the sensor is dependent on the melt pool's surface angle. Measured intensity is reduced at the edges of the melt pool due to higher surface angles. Higher emission levels are measured from the substrate due to low surface angles. This is referred to as the directional emittance phenomenon throughout this paper and results in images with true melt pool edge features. Figure 2 is a schematic representation of the emission theory.

a)

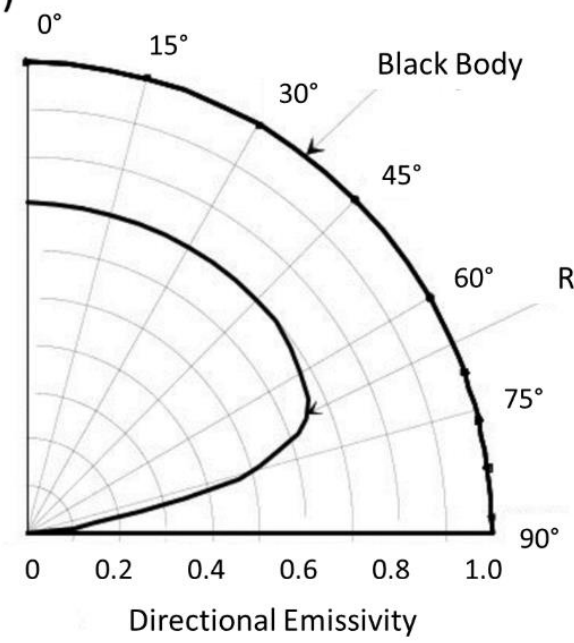

b)

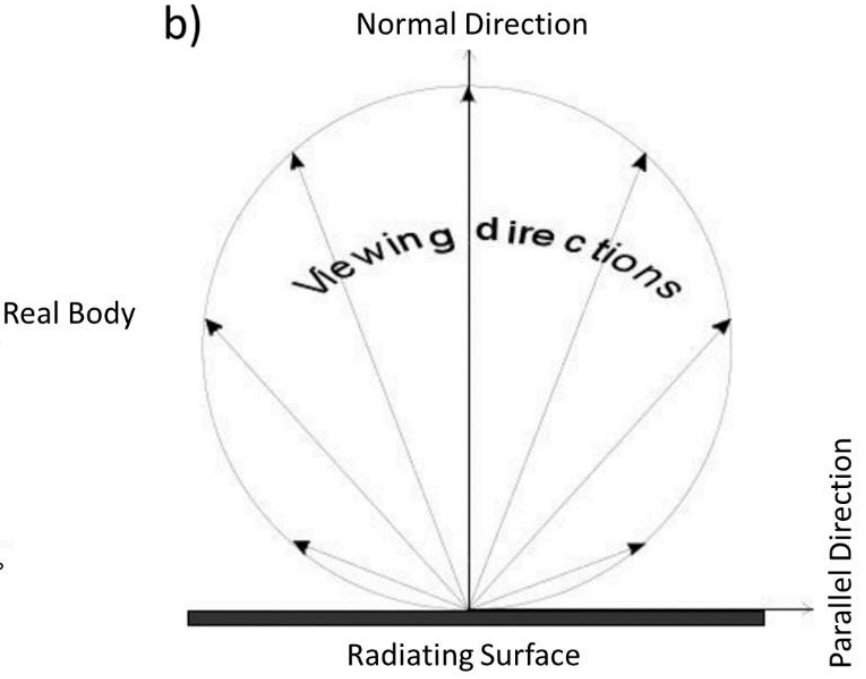

Figure 2. a) Directional emittance of a blackbody and real body. b) A schematic representation of directional emittance from a flat surface. Documented by Meola (2016).

\section{Melt Pool Width Extraction}

The melt pool image processing algorithm used in this study was discussed previously by Sampson et al. (2020). The image processing algorithm uses the directional emittance phenomena and optimised melt pool images to extract melt pool width measurements. The contrast between the melt pool edge and brightly lit substrate provides a unique feature that can be extracted using image processing techniques. Cross-sectional signals are extracted, averaged and differentiated to expose edge locations. Locations of the melt pool's edges are then used to calculate melt pool width. Figure 3 displays melt pool width calculations using the directional emittance technique. 


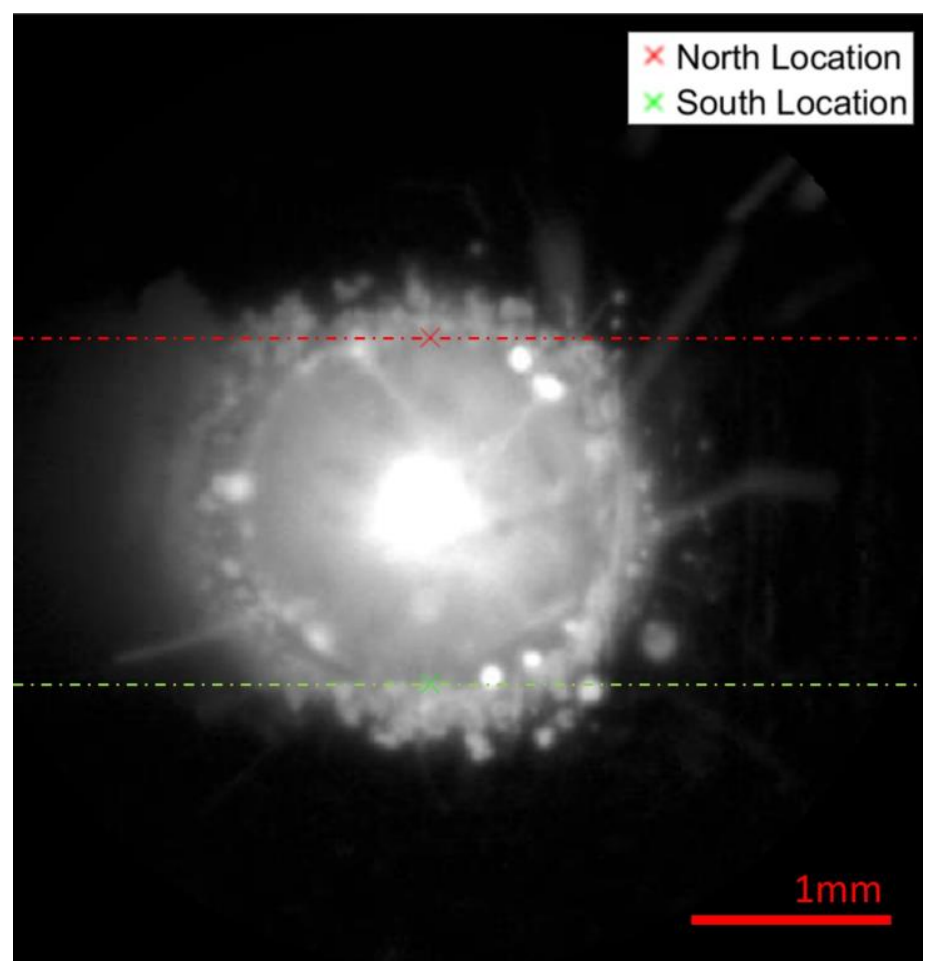

Figure 3. An image showing the north and south locations calculated using the directional emittance technique.

\section{Melt Pool Width Track Averages}

To directly compare width measurement signals (extracted from melt pool video recordings of different tracks) for parametric studies, snippets are extracted from the original full track deposition data. Taking snippets from recordings allows for an easier comparison as fluctuations in the acceleration/stabilisation and deceleration/destabilisation stages of deposition are eradicated.

For data collection, snippet lengths are half that of the original signals. The deposition start point is determined when the first melt pool measurement was recorded for each individual track. The starting point for snippet extraction began after $25 \%$ of the track length had passed and finished before the last $25 \%$ of the track length. This resulted in signals that were not clouded by the unstable nature of the melt pool in the acceleration/stabilisation and deceleration/destabilisation stages of deposition. Figure 4 presents an original melt pool signal, with guidelines to display the extraction process. The frame numbers on the extracted signal are not carried over from the original signal and are reset. 


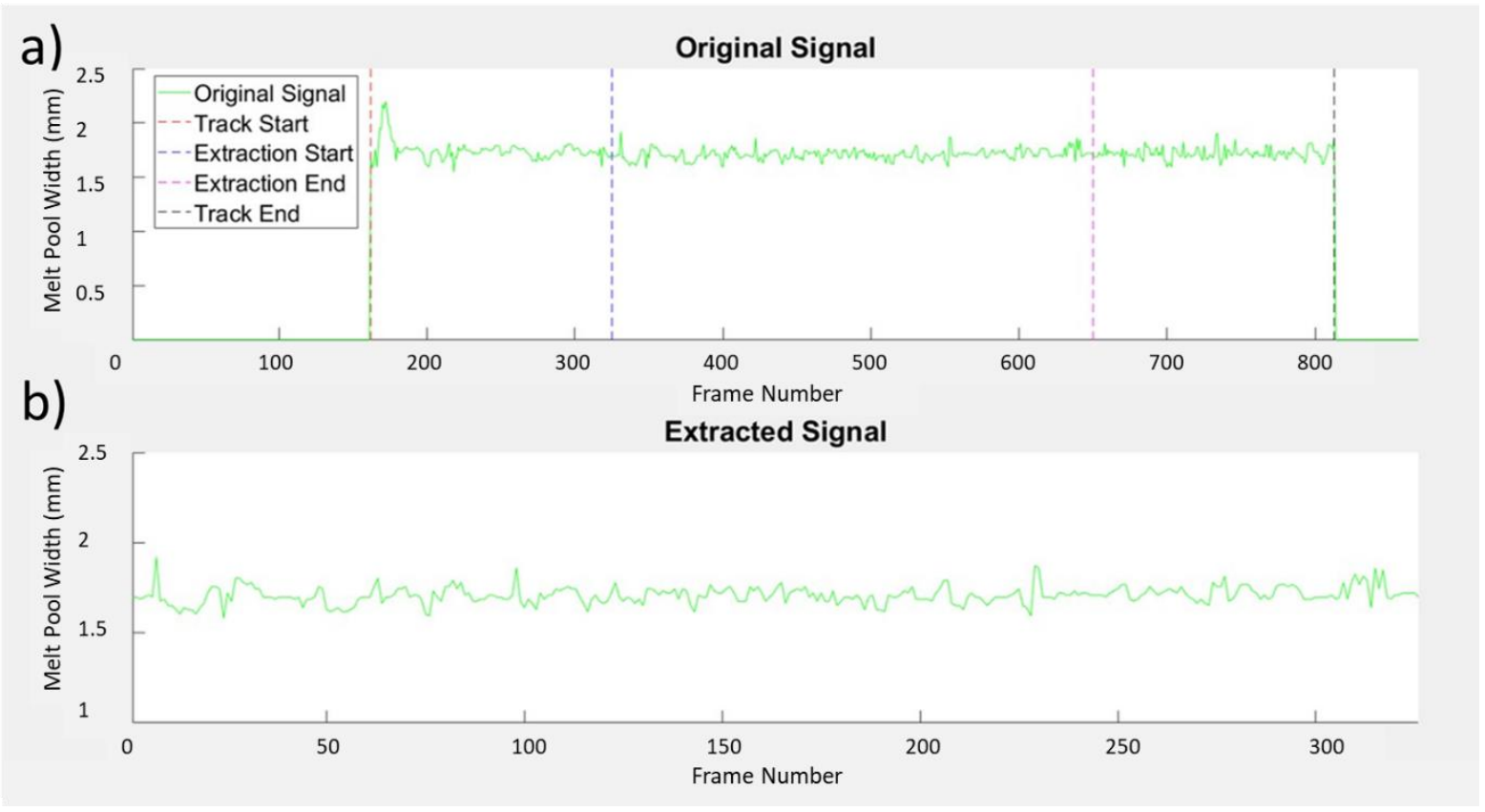

Figure 4. a) An original signal of the melt pool width calculations for a recording of a track deposition. b) The extracted signal used for melt pool dimensioning comparison.

\section{Experimental Configuration}

\section{Equipment Set-up}

In this research a Trumpf Trudisk 8002 5.3kW disc laser DED system was used with a TruControl 1000 controller. Trumpf BEO D70 processing optics with a motorised collimation laser deposition head were employed with a Reis RV60-40 robot, a Reis RDKVv05 two axis manipulator and a Sulzer Metco 10$\mathrm{C}$ powder feeder with a dual $1.5 \mathrm{~kg}$ hopper arrangement.

A NIR CMOS machine vision camera (U3-3240ML-NIR-GL) was installed to improve melt pool imaging and was combined with a UV/VIS cut off imaging filter with a $135 \mathrm{~nm}$ notch. The filter had an optical density of 3.0 for wavelengths of $200-750 \mathrm{~nm}$ and 4.0 for wavelengths of $1000-1200 \mathrm{~nm}$. The notch filter and NIR CMOS camera were coaxially installed into the laser deposition head for a clear birds-eye view of the melt pool.

\section{Material}

The deposition material for this study was the steel alloy EN25. The composition is presented in Table 1. It should be noted that powder from different manufacturers and even different batches of powder can cause changes in melt pool characteristics.

Table 1. The chemical composition of the EN25 powder used for experimental deposition (wt\%)

\begin{tabular}{ccccccccc}
$\mathrm{Al}$ & $\mathrm{Cr}$ & $\mathrm{Cu}$ & $\mathrm{Mn}$ & $\mathrm{Mo}$ & $\mathrm{Ni}$ & $\mathrm{P}$ & $\mathrm{Si}$ & $\mathrm{C}$ \\
\hline 0.003 & 0.69 & 0.006 & 0.64 & 0.59 & 2.66 & 0.004 & 0.26 & 0.32 \\
$\mathrm{~S}$ & $\mathrm{O}$ & $\mathrm{N}$ & $\mathrm{Fe}$ & $\mathrm{TAO}$ & $\mathrm{B}$ & $\mathrm{Mg}$ & $\mathrm{Zr}$ & $\mathrm{Co}$ \\
\hline 0.003 & 0.02 & 0.003 & $\mathrm{Bal}$ & 0.01 & $<0.001$ & $<0.001$ & $<0.01$ & 0.01
\end{tabular}




\section{Method}

Single line tracks with lengths of $150 \mathrm{~mm}$ were deposited onto a $15 \mathrm{~mm}$ thick stainless steel $316 \mathrm{~L}$ substrate plate for all experiments. Two separate parametric experiments were conducted, each consisting of 45 cladding tracks. Substrate temperatures were measured before each cladding track was deposited to ensure that heat accumulation did not affect results. Cladding tracks were deposited with enough distance separation so that previous tracks did not interfere with further deposition. Figure 5. A schematic representation of the cladding tracks deposited on the stainless-steel plate substratesFigure 5 displays schematically how tracks were deposited. The direction of travel was fixed, the carrier gas was set to $3.5 \mathrm{~L} / \mathrm{min}$, the shielding gas was set to $6 \mathrm{~L} / \mathrm{min}$, the laser spot size was $1.5 \mathrm{~mm}$, and three laser power settings of 750,900 and $1050 \mathrm{~W}$ were used. All these settings remained consistent throughout the experiment. Two separate trials were conducted, to determine the influence of both PMFR and path velocity on melt pool width. The PMFR setting was iterated from $3.70-5.29 \mathrm{~g} / \mathrm{min}$. PMFR is controlled by an opening on the hopper, with a percentage value indicating how much the hopper is open. This value was changed from 9 to $12.5 \%$ on the machine using 15 increments of $0.25 \%$ to achieve the described PMFR. The path velocity setting was iterated from 9.5 to $13 \mathrm{~mm} / \mathrm{s}$ with a total of 15 increments $(0.25 \mathrm{~mm} / \mathrm{s})$. The matrices of parameters used for the experiments are displayed in Table 2 and

Table 3. All values were selected within the parameter envelope of EN25 and are known to produce quality tracks with good mechanical properties and reduced defects. Parameters outside these ranges are known to produce poor quality deposition for EN25.

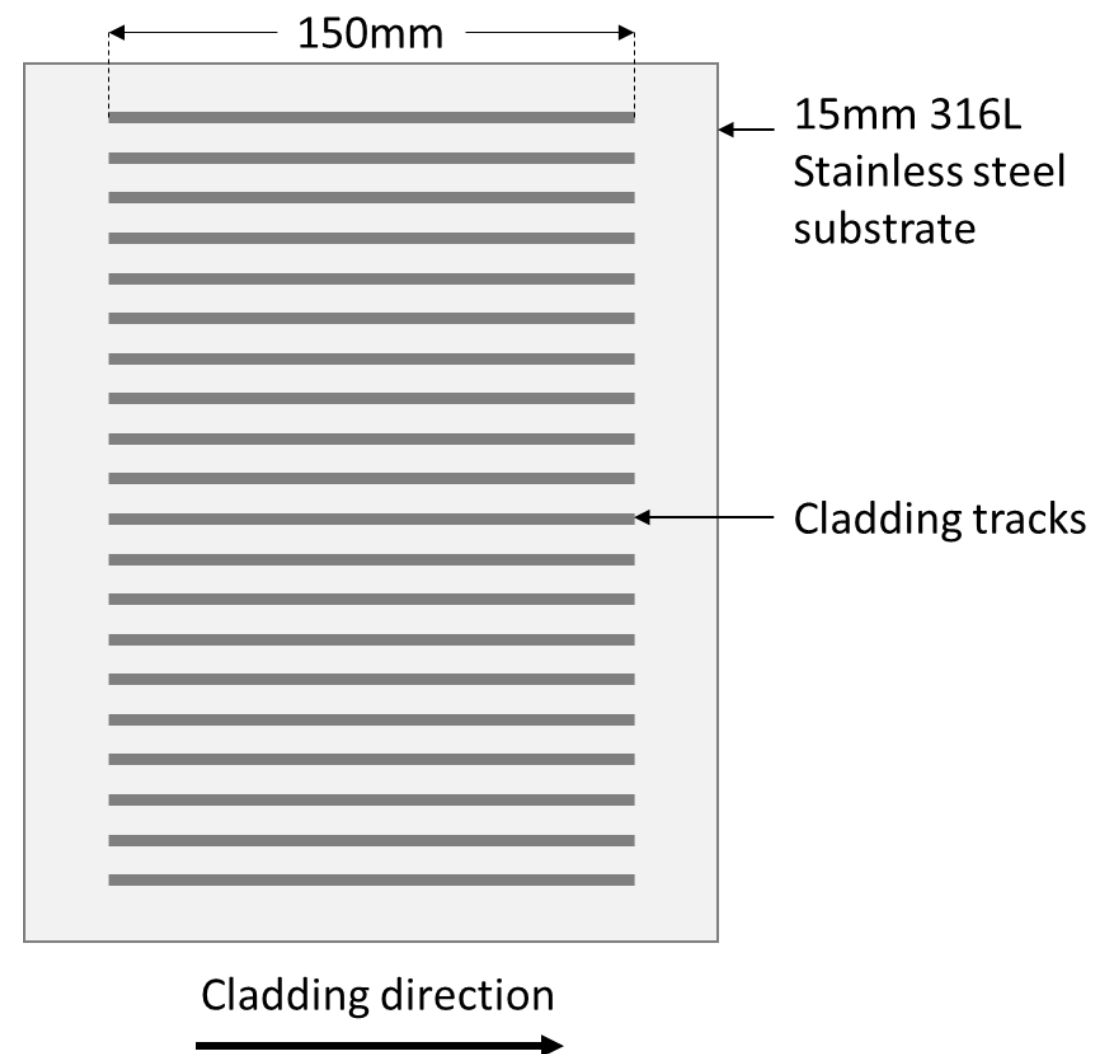

Figure 5. A schematic representation of the cladding tracks deposited on the stainless-steel plate substrates 
Table 2. The matrix of parameters used in the PMFR experiment

\begin{tabular}{|c|c|c|c|c|c|}
\hline $\begin{array}{c}\text { Laser } \\
\text { Power } \\
(\mathrm{W})\end{array}$ & $\begin{array}{l}\text { PMFR } \\
(\mathrm{g} / \mathrm{min})\end{array}$ & $\begin{array}{c}\text { Laser Power } \\
\text { (W) }\end{array}$ & $\begin{array}{l}\text { PMFR } \\
\text { (g/min) }\end{array}$ & $\begin{array}{l}\text { Laser Power } \\
\text { (W) }\end{array}$ & $\begin{array}{l}\text { PMFR } \\
\text { (g/min) }\end{array}$ \\
\hline 750 & 3.70 & 900 & 3.70 & 1050 & 3.70 \\
\hline 750 & 3.81 & 900 & 3.81 & 1050 & 3.81 \\
\hline 750 & 3.93 & 900 & 3.93 & 1050 & 3.93 \\
\hline 750 & 4.04 & 900 & 4.04 & 1050 & 4.04 \\
\hline 750 & 4.15 & 900 & 4.15 & 1050 & 4.15 \\
\hline 750 & 4.27 & 900 & 4.27 & 1050 & 4.27 \\
\hline 750 & 4.38 & 900 & 4.38 & 1050 & 4.38 \\
\hline 750 & 4.49 & 900 & 4.49 & 1050 & 4.49 \\
\hline 750 & 4.61 & 900 & 4.61 & 1050 & 4.61 \\
\hline 750 & 4.72 & 900 & 4.72 & 1050 & 4.72 \\
\hline 750 & 4.83 & 900 & 4.83 & 1050 & 4.83 \\
\hline 750 & 4.95 & 900 & 4.95 & 1050 & 4.95 \\
\hline 750 & 5.06 & 900 & 5.06 & 1050 & 5.06 \\
\hline 750 & 5.17 & 900 & 5.17 & 1050 & 5.17 \\
\hline 750 & 5.29 & 900 & 5.29 & 1050 & 5.29 \\
\hline
\end{tabular}

Table 3. The matrix of parameters used in the path velocity experiment

\begin{tabular}{|c|c|c|c|c|c|}
\hline $\begin{array}{c}\text { Laser } \\
\text { Power } \\
(\mathrm{W})\end{array}$ & $\begin{array}{c}\text { Path } \\
\text { Vel. } \\
(\mathrm{mm} / \mathrm{s})\end{array}$ & $\begin{array}{l}\text { Laser Power } \\
\text { (W) }\end{array}$ & $\begin{array}{c}\text { Path } \\
\text { Vel. } \\
(\mathrm{mm} / \mathrm{s})\end{array}$ & $\begin{array}{l}\text { Laser Power } \\
\text { (W) }\end{array}$ & $\begin{array}{c}\text { Path } \\
\text { Vel. } \\
(\mathrm{mm} / \mathrm{s})\end{array}$ \\
\hline 750 & 9.5 & 900 & 9.5 & 1050 & 9.5 \\
\hline 750 & 9.75 & 900 & 9.75 & 1050 & 9.75 \\
\hline 750 & 10 & 900 & 10 & 1050 & 10 \\
\hline 750 & 10.25 & 900 & 10.25 & 1050 & 10.25 \\
\hline 750 & 10.5 & 900 & 10.5 & 1050 & 10.5 \\
\hline 750 & 10.75 & 900 & 10.75 & 1050 & 10.75 \\
\hline 750 & 11 & 900 & 11 & 1050 & 11 \\
\hline 750 & 11.25 & 900 & 11.25 & 1050 & 11.25 \\
\hline 750 & 11.5 & 900 & 11.5 & 1050 & 11.5 \\
\hline 750 & 11.75 & 900 & 11.75 & 1050 & 11.75 \\
\hline 750 & 12 & 900 & 12 & 1050 & 12 \\
\hline 750 & 12.25 & 900 & 12.25 & 1050 & 12.25 \\
\hline 750 & 12.5 & 900 & 12.5 & 1050 & 12.5 \\
\hline 750 & 12.75 & 900 & 12.75 & 1050 & 12.75 \\
\hline 750 & 13 & 900 & 13 & 1050 & 13 \\
\hline
\end{tabular}

Using standard recording software (distributed with the U3-3240ML-NIR-GL camera), a total of 90 videos were recorded for the entire track lengths. The melt pool width average for each track was extracted from the corresponding videos in the manner described within this paper.

\section{Results}


This section is presented in two separate subsections, describing results for the PMFR and path velocity experiments separately. Each experiment consisted of 45 individual cladding tracks, which were deposited on substrates as depicted in Figure 5. The large amount of data collected has been collated into single graphs for each experiment to allow for comprehensive analysis. Figure 6 and 7 represent the coalesced data for the PMFR and path velocity experiments respectively. Each data point on the graph represents a melt pool width average for a single clad track, calculated using the melt pool width extraction and track averaging technique outlined within this paper.

\section{Powder Mass Flow Rate}

Results from the PMFR parametric experiment detailed in Table 2 were plotted on a single graph, as depicted in Figure 6.

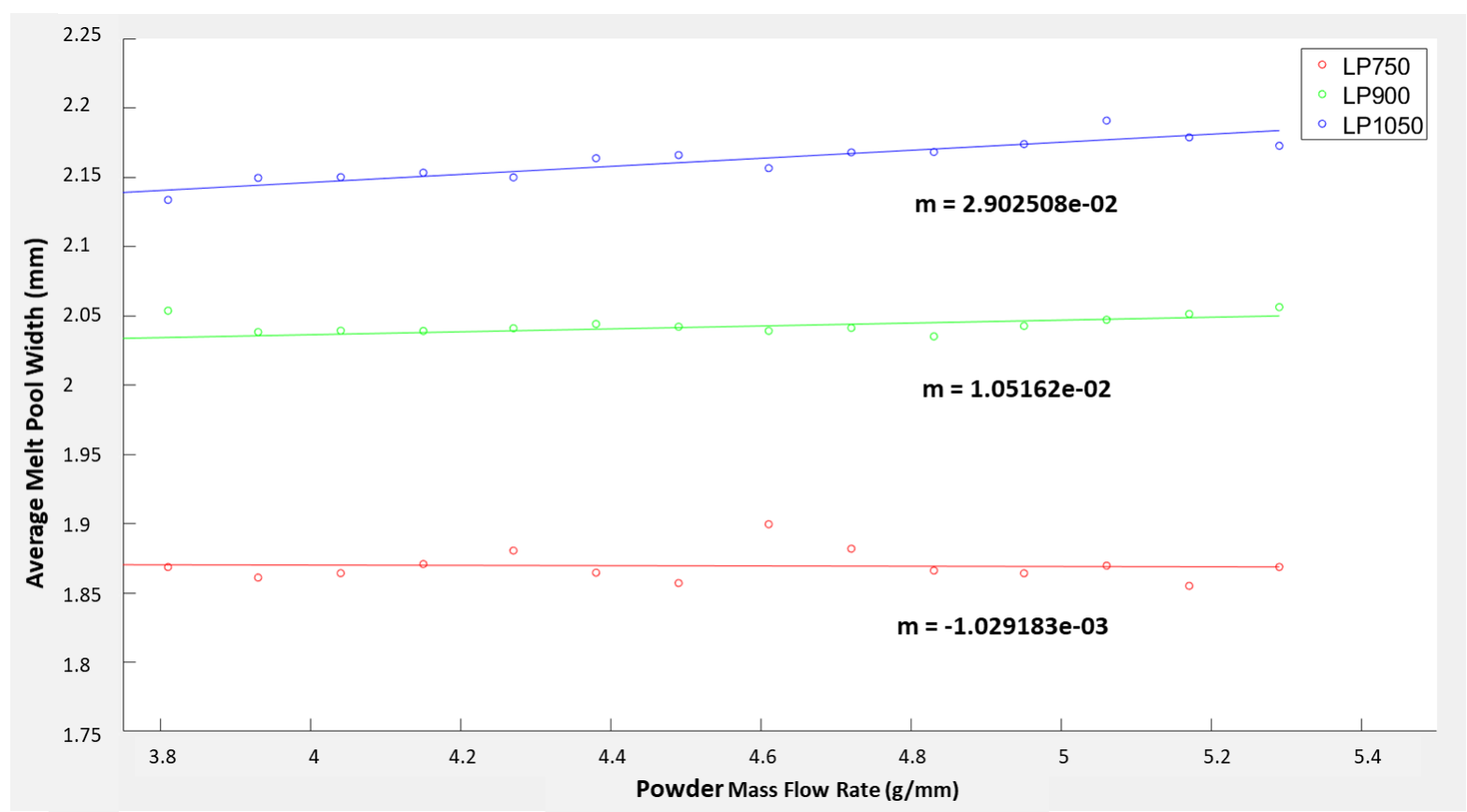

Figure 6. Graph representing all calculated average melt pool values for various parameter settings used in the PMFR experiment.

The results have shown that the effects of changing PMFR on the melt pool width is dependent on the laser power, and it cannot simply be assumed that the melt pool width increases with increasing PMFR. For the highest laser power setting used in this experiment $(1050 \mathrm{~W})$ the melt pool width did increase when an increasing PMFR was used. Having a high laser power setting means that there is more energy to melt powder and increasing the PMFR in this scenario resulted in more powder being absorbed into the melt pool. This in turn led to an increase in melt pool width.

For the mid (900W) laser power setting this was again true. Having a relatively high laser power setting resulted in more powder being melted and accepted into the molten melt pool when the PMFR was increased.

The gradients of the lines for both the mid and high laser power settings differ. The tracks with a mid laser power setting showed a shallower increase in melt pool width for the same increase in PMFR. This is most likely due to there being insufficient energy to melt excess powder as effectively.

The gradient of the line of best fit for the low (750W) laser power setting is close to zero. Results showed that increasing the PMFR for a low laser power setting did not increase the size of the melt pool. The average melt pool width stayed constant as the PMFR was increased. For tracks deposited 
using the lower laser power, there is larger deviation of calculated melt pool width values from the line of best fit. Using a low laser power setting showed more variance between melt pool width calculations which indicated melt pool instability.

Results have indicated that increasing PMFR does not always increase melt pool width. This behaviour cannot be assumed for all parameter settings. The effects that increasing/decreasing the PMFR has on the melt pool is dependent on other parameter settings used.

\section{Path Velocity}

Results from the path velocity parametric experiment described in Table 3 were plotted on a single graph, as depicted in Figure 7.

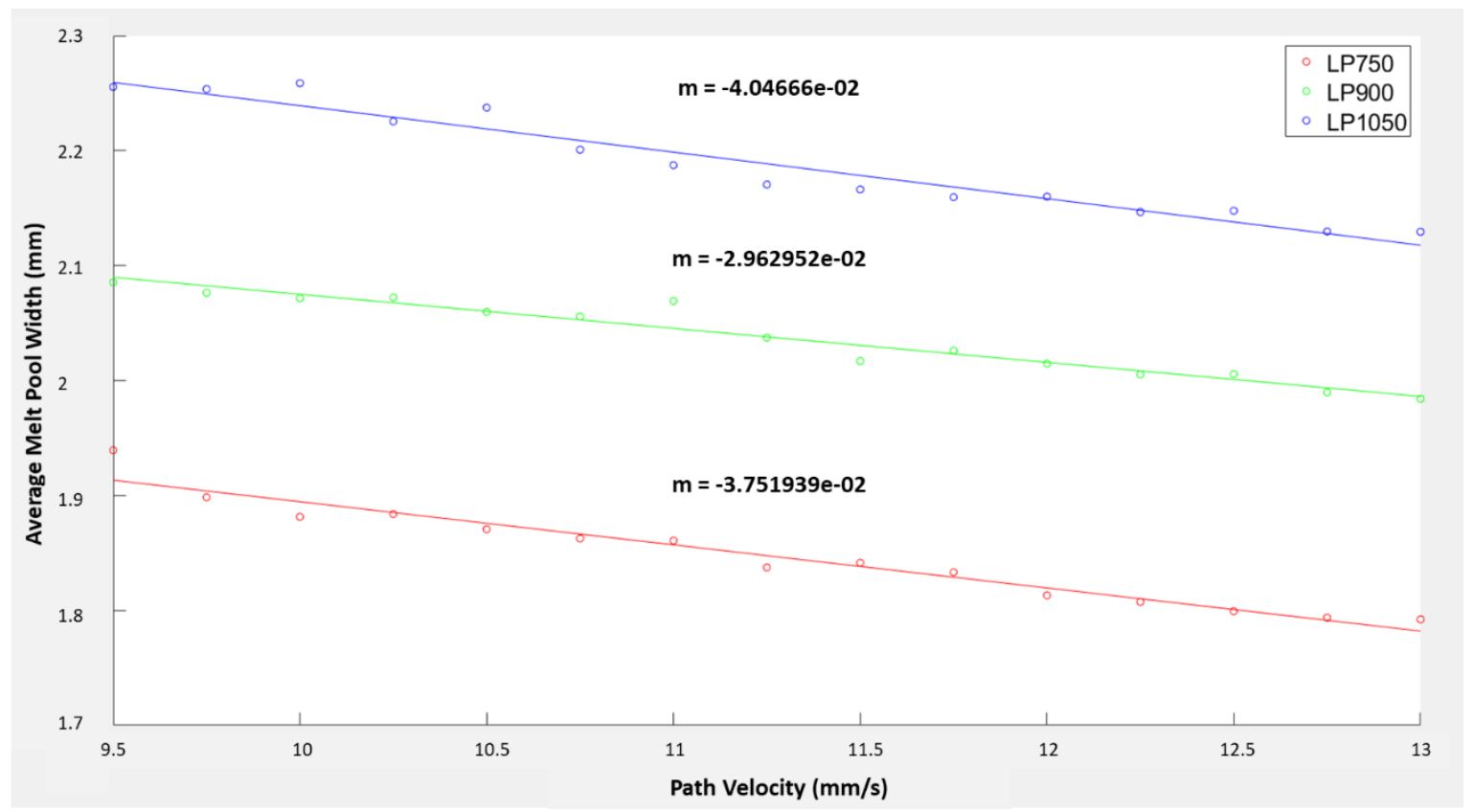

Figure 7. A graph representing all calculated average melt pool values for various parameter settings used in the path velocity experiment.

The results from this plot provide information regarding the influence that the path velocity setting has on calculated melt pool widths. For all laser power settings, the average melt pool width decreased when the path velocity was increased. The melt pool width increases when the laser power setting is increased due to a higher energy density melting more blown powder and substrate material.

Increasing the path velocity results in a decrease in energy density, which in turn results in less energy being available to melt the molten powder and substrate in an instance. In addition, higher translation speeds reduce the interaction time between the laser and powder, resulting in less powder absorption into the melt pool. Both factors likely result in a reduction of melt pool width. The gradients of the three lines of best fit have also been displayed on the graph. The lines of best fit all show a negative gradient of similar values. This said, performing the cladding tracks at a laser power of $900 \mathrm{~W}$ produced the shallowest gradient. The difference between this gradient and the other two could be a result of the complex interactions that occur between different parameters. Increasing the path velocity could have different results on the melt pool geometry depending on other parameters that are used in the process. However, before conclusions are drawn more research should be performed to further increase understanding. 


\section{Discussion}

This research aimed to provide a detailed understanding of how changes in AM parameters can affect melt pool geometry. Studies have shown that PMFR is a parameter known to greatly affect DED processes, with Meriaudeau et al. (1997) and Yao et al. (2018) concluding that it majorly influences both melt pool behaviour and part quality. This research has highlighted that changes in PMFR greatly affects melt pool width, and that these changes can be accurately measured using the directional emittance technique outlined be Sampson et al. (2020). This research extends knowledge contributed by Meriaudeau et al. (1997) and Yao et al. (2018) by providing a detailed study on the interaction between melt pool width, laser power and PMFR. This information can provide users with greater process understanding, which can subsequently lead to higher quality deposition.

Sun \& Hao (2012) and Meriaudeau et al. (1997) both explored the effects of PMFR on solidified track dimensions and stated that PMFR is one of the dominant features affecting these. Their research shows that increasing the PMFR simply increases the size of the track width, but they do not detail the size of the melt pool throughout the process. Results from this paper detail the effects of changing PMFR on melt pool width and expose a complex relationship. Although melt pool width and track width are positively correlated, there are subtle differences between these dimensions due to shrinkage in material during solidification. Previous research from Sampson et al. (2020) shows that both melt pool and track width increase with increasing laser power. They do however highlight that changes in shrinkage rates are apparent for different laser power settings. Sampson et al. (2020) conclude that whilst melt pool width and track width are related, shrinkage rates are influenced by laser power and likely other parameters. Future work should look to explore the complex relationships between melt pool width and track width for varying PMFR settings.

Meriaudeau et al. (1997) details the effects that path velocity has on solidified track width and reports that increasing path velocity results in a decrease in track width. Although this study measures the effects of path velocity on solidified track width, melt pool width and track width are undoubtedly linked. Previous work from Sampson et al. (2020) has shown that whilst shrinkage rates can change for different parameters, the melt pool width and track width are positively correlated. Results for the path velocity experiment within this paper compliment those that were published by Meriaudeau et al. (1997).

Hua et al. (2008) details the effects that path velocity has on melt pool temperature and layer thickness. Their results show that increasing the path velocity reduces the temperature of the melt pool during manufacturing. Comparing results shows that increasing path velocity reduces both the melt pool width and temperature. This compliments the statement that increasing path velocity reduces the energy density for a specific instance, resulting in lower temperature melt pools and less powder being able to be absorbed.

Cheng et al. (2018) detailed the effects of path velocity on melt pool width and indicated that increasing path velocity results in a decrease in melt pool width. The experiments conducted by Cheng et al. (2018) were performed on a PBF machine using Inconel 718 powder. Despite differences in both the AM technique and material used, the same results were evident within this paper.

This paper demonstrates that the directional emittance algorithm can be used to effectively study parameter interaction. Results have provided a deeper understanding of the interaction between PMFR and melt pool width for multiple laser power settings. This research has solidified the understanding that increasing path velocity decreases melt pool width. Future work should look to further study the relationship between melt pool width, temperature and solidified track measurements, for various parameter settings. Having a deeper understanding of the influences of parameters on both the melt pool and formed track will help develop advanced material understanding and improve build quality.

\section{Conclusion}


This paper implemented a previously published directional emittance image processing technique to further understand process parameter interactions in DED processes. Two separate parameter trials were conducted to determine how both the PMFR and path velocity influence the melt pool's width.

Increasing the PMFR when there is excess energy available (using higher laser power settings) results in the melt pool width increasing as more powder is melted by the excess energy. When using high laser power settings $(1050 \mathrm{~W})$, a larger body of molten material was formed. This was evident on the mid laser power settings $(900 \mathrm{~W})$, but the influence that increasing PMFR had on the melt pool width was reduced. Deposition using a low laser power setting $(750 \mathrm{~W})$ showed results that have not been documented previously and increasing the PMFR at this laser power setting resulted in no increase in melt pool width. Low energy levels were not able to melt the extra powder that was introduced and resulted in the melt pool not growing.

Increasing the path velocity showed a decrease in melt pool width for all laser power settings. Some variances in laser power settings resulted in changes to the rate of melt pool width decreasing, but the differences were not as prominent as those displayed in the PMFR study. No conclusion could be drawn from the three laser power settings used in this study and the subtle differences between them. The conclusion drawn from this experiment is that increasing path velocity results in the melt pool width decreasing. This compliments previous findings.

The directional emittance algorithm has been successfully used to better understand the complex DED process and has uncovered how certain parameters influence the melt pool in more detail. Results show that laser power, PMFR and path velocity have complex interactions and changing one can directly influence how the melt pool reacts to further parameter changes. The directional emittance algorithm has been successfully used to perform a detailed parametric study.

\section{Acknowledgements}

This research was funded through the National Research Network in Advanced Engineering and Materials (NRN167) and TWI, United Kingdom.

\section{Data Availability Statement}

The raw/processed data required to reproduce these findings cannot be shared at this time as the data also forms part of an ongoing study.

\section{References}

Cheng, B., Lydon, J., Cooper, K., Cole, V., Northrop, P., \& Chou, K. (2018). Melt pool sensing and size analysis in laser powder-bed metal additive manufacturing. Journal of Manufacturing Processes, 32(November 2017), 744-753. https://doi.org/10.1016/j.jmapro.2018.04.002

Colodrón, P., Fariña, J., Rodríguez-Andina, J. J., Vidal, F., Mato, J. L., \& Montealegre, Á. (2011a). Performance improvement of a laser cladding system through FPGA-based control. IECON $\begin{array}{llll}\text { Proceedings } \quad \text { (Industrial Electronics } & \text { Conference), } & \text { 2814-2819. }\end{array}$ https://doi.org/10.1109/IECON.2011.6119758

Colodrón, P., Fariña, J., Rodríguez-Andina, J. J., Vidal, F., Mato, J. L., \& Montealegre, M. Á. (2011b). FPGA-based measurement of melt pool size in laser cladding systems. Proceedings - ISIE 2011: 2011 IEEE International Symposium on Industrial Electronics, 1503-1508. https://doi.org/10.1109/ISIE.2011.5984383

Craeghs, T., Clijsters, S., Kruth, J.-P., Bechmann, F., \& Ebert, M.-C. (2012). Detection of Process Failures in Layerwise Laser Melting with Optical Process Monitoring. Physics Procedia, 39, $753-$ 759. https://doi.org/10.1016/j.phpro.2012.10.097

Dai, D., Gu, D., Zhang, H., Xiong, J., Ma, C., Hong, C., \& Poprawe, R. (2018). Influence of scan strategy and molten pool configuration on microstructures and tensile properties of selective laser melting additive manufactured aluminum based parts. Optics and Laser Technology, 99, 91-100. 
https://doi.org/10.1016/j.optlastec.2017.08.015

Ding, X., Koizumi, Y., Wei, D., \& Chiba, A. (2019). Effect of process parameters on melt pool geometry and microstructure development for electron beam melting of IN718: A systematic single bead analysis study. Additive Manufacturing, 26(December 2018), 215-226. https://doi.org/10.1016/j.addma.2018.12.018

Fiegl, T., Franke, M., \& Körner, C. (2019). Impact of build envelope on the properties of additive manufactured parts from AlSi10Mg. Optics and Laser Technology, 111(June 2018), 51-57. https://doi.org/10.1016/j.optlastec.2018.08.050

Furumoto, T., Egashira, K., Munekage, K., \& Abe, S. (2018). Experimental investigation of melt pool behaviour during selective laser melting by high speed imaging. CIRP Annals, 67(1), 253-256. https://doi.org/10.1016/j.cirp.2018.04.097

Grevey, F. M. F. T. D., \& Vannes, a B. (1997). Laser Cladding process and image processing. Journal of Laser in Engineering, 6(33), 161-187.

Hofman, J. T., Pathiraj, B., van Dijk, J., de Lange, D. F., \& Meijer, J. (2012). A camera based feedback control strategy for the laser cladding process. Journal of Materials Processing Technology, 212(11), 2455-2462. https://doi.org/10.1016/j.jmatprotec.2012.06.027

Hooper, P. A. (2018). Melt pool temperature and cooling rates in laser powder bed fusion. Additive Manufacturing, 22(May), 548-559. https://doi.org/10.1016/j.addma.2018.05.032

Hua, T., Jing, C., Xin, L., Fengying, Z., \& Weidong, H. (2008). Research on molten pool temperature in the process of laser rapid forming. Journal of Materials Processing Technology, 198(1-3), 454462. https://doi.org/10.1016/j.jmatprotec.2007.06.090

Kledwig, C., Perfahl, H., Reisacher, M., Brückner, F., Bliedtner, J., \& Leyens, C. (2019). Analysis of melt pool characteristics and process parameters using a coaxial monitoring system during directed energy deposition in additive manufacturing. Materials, 12(2). https://doi.org/10.3390/ma12020308

Manvatkar, V., De, A., \& DebRoy, T. (2015). Spatial variation of melt pool geometry, peak temperature and solidification parameters during laser assisted additive manufacturing process. Materials Science and Technology, 31(8), 924-930. https://doi.org/10.1179/1743284714Y.0000000701

Meola, C. (2016). Infrared Thermography: Recent Advances And Future Trends. (January).

Meriaudeau, F., \& Truchetet, F. (1996). Control and optimization of the laser cladding process using matrix cameras and image processing. Journal of Laser Applications, 8, 317-324. https://doi.org/10.2351/1.4745438

Renier, E., Meriaudeau, F., \& Truchetet, F. (1996). CCD Technology Applied To Industrial Welding Applications. Melecon_96, 1335-1338.

Sampson, R., Lancaster, R., Sutcliffe, M., Carswell, D., Hauser, C., \& Barras, J. (2020). An improved methodology of melt pool monitoring of direct energy deposition processes. Optics and Laser Technology, 127(December 2019). https://doi.org/10.1016/j.optlastec.2020.106194

Sun, Y., \& Hao, M. (2012). Statistical analysis and optimization of process parameters in Ti6Al4V laser cladding using Nd:YAG laser. Optics and Lasers in Engineering, 50(7), 985-995. https://doi.org/10.1016/j.optlaseng.2012.01.018

Sun, Z., Guo, W., \& Li, L. (2020). In-process measurement of melt pool cross-sectional geometry and grain orientation in a laser directed energy deposition additive manufacturing process. Optics and Laser Technology, 129(April), 106280. https://doi.org/10.1016/j.optlastec.2020.106280

Yan, Z., Liu, W., Tang, Z., Liu, X., Zhang, N., Li, M., \& Zhang, H. (2018). Review on thermal analysis in laser-based additive manufacturing. Optics and Laser Technology, 106, 427-441. https://doi.org/10.1016/j.optlastec.2018.04.034

Yao, Y., Huang, Y., Chen, B., Tan, C., Su, Y., \& Feng, J. (2018). Influence of processing parameters and heat treatment on the mechanical properties of $18 \mathrm{Ni} 300$ manufactured by laser based directed energy deposition. Optics and Laser Technology, 105, 171-179. https://doi.org/10.1016/j.optlastec.2018.03.011 